\title{
Fecal Microbiome Transplantation for Recurrent Clostridioides difficile Infection: Treatment Efficacy, Short and Long-term Follow-up Results from Consecutive Case Series
}

Tadas Urbonas $^{1,2^{\star}}$, Gianluca Ianiro ${ }^{3^{*}}$, Rolandas Gedgaudas ${ }^{1,2}$, Povilas Sabanas ${ }^{1,2}$, Mindaugas Urba $^{1,2}$, Vytautas Kiudelis $^{1,2}$, Gediminas Kiudelis $^{1,2}$, Vytenis Petkevicius ${ }^{1,2}$, Astra Vitkauskiene ${ }^{4}$, Giovanni Cammarota ${ }^{3}$, Antonio Gasbarrini ${ }^{3}$, Juozas Kupcinskas $^{1,2}$

1) Department of Gastroenterology, Lithuanian University of Health Sciences, Kaunas, Lithuania;

2) Institute for Digestive

Research, Lithuanian

University of Health Sciences,

Kaunas, Lithuania;

3) Digestive Disease Center, Fondazione Policlinico Universitario A. Gemelli IRCCS, Università Cattolica Del Sacro Cuore, Rome, Italy; 4) Departament of Laboratory Medicine, Lithuannian University of Health Science, Kaunas, Lithuania

Address for correspondence: Tadas Urbonas Department of Gastroenterology and Institute for Digestive Research, Lithuanian University of Health Sciences, Eivenių str. 2, LT-50009, Kaunas, Lithuania. tadas.urbonas@lsmuni.lt

Received: 31.05.2021 Accepted: 05.09.2021

* Equal contribution

\section{ABSTRACT}

Background \& Aims: Many studies have shown a high effectiveness of fecal microbiota transplantation (FMT) in treatment of recurrent or refractory Clostridioides difficile infection (CDI). Nevertheless, data on long term outcomes and complications after FMT are still lacking. We aimed to evaluate the efficacy, the periprocedural safety profile and the long-term efficacy and safety of FMT for recurrent CDI during a median follow up period of 24 months.

Methods: Our study included 60 consecutive patients that were treated from 2015 to 2019 for recurrent CDI. In all patients FMT was performed through the nasoenteric tube placed during gastroscopy. Fresh donor feces were used for FMT from unrelated donors. Pre-FMT preparation included CDI treatment with oral vancomycin $500 \mathrm{mg}$ q.i.d. for at least five days and proton pump inhibitor (PPI) administration before FMT. Follow up data included information about recurrent CDI episodes, early and late complications, health status at 3, 12 and 24 months after FMT.

Results: FMT was performed in 60 patients (median age 72.5 years) with recurrent CDI. Clinical improvement after the first FMT procedure was observed in 48 patients (80\%). Ten of 12 initially non-responding patients had a clinical resolution after a second FMT leading to an increased overall cure rate of $96.7 \%$. The remaining two patients needed a third FMT with a final overall cure rate of $100 \%$. Nine of 60 patients were under immunosuppressive therapy. Six immunosuppressed patients were in the group of initial responders and the remaining three in the initially non-responder group. We observed a very low rate of adverse events in the short and long-term follow-up after FMT. During the first eight weeks after the FMT procedure, the death of three patients occurred, but they were not related to the FMT procedure. Patients were followed up for a median of 20 months, with the range from 12 to 55 months. During the follow-up period no long-term serious adverse events (SAE) were documented.

Conclusions: Our study confirms excellent efficacy rates of FMT in the treatment of recurrent CDI. In addition, this study shows that it is possible to avoid short term SAE when FMT is administered via a nasoenteric tube by following a very stringent peri-procedural patient follow-up protocol. Our study also demonstrates good safety with a low rate of long-term adverse events after FMT.

Key words: fecal microbiota transplantation - Clostridioides difficile - follow-up - adverse events immunosuppression.

Abbreviations: CDI: Clostridioides difficile infection; FMT: fecal microbiota transplantation; PPI: proton pump inhibitor; SAE: serious adverse effect.

\section{INTRODUCTION}

Clinical manifestations of Clostridioides difficile infection (CDI) range from mild to very severe enterocolitis. Older age, immunosuppression, chemotherapy and chronic kidney diseases are well recognized risk factors for CDI and unfavorable outcomes of the infection $[1,2]$. First episodes of CDI infection are usually managed by antibiotics and current guidelines recommend using oral vancomycin as the first line treatment $[3,4]$. Nevertheless, CDI tends to reoccur in a significant proportion of patients and in the most severe cases intractable enterocolitis may even result in fatal outcomes $[5,6]$.

Over the last years fecal microbiome transplantation (FMT) has emerged as a very effective treatment option for 
recurrent or refractory CDI [4, 7-11]. The efficacy of FMT for the management of recurrent CDI has been proven by a recent meta-analysis $[7,12]$. Furthermore, emerging evidence suggests that FMT should be considered for patients with severe and fulminant CDI refractory to antibiotic therapy [13]. Fecal microbiome transplantation has been shown to reduce health expenditure [14] and improve the quality of life. More recently, FMT has been extensively studied in a number of different indications including inflammatory bowel diseases [15-18], irritable bowel syndrome [19-21], hepatic encephalopathy $[22,23]$, tyrosine-kinase inhibitors induced diarrhea [24] and other clinical conditions [25-27], although CDI still remains the only evidence-based indication in clinical practice $[28,29]$.

Fecal microbiome transplantation procedure is considered to be safe with a few serious adverse events (SAEs) [30, 31]. Most of FMT adverse events are minor (abdominal pain, discomfort, bloating, flatulence) and SAEs like high-grade fever, infection, sepsis, pneumonia, endoscopy related adverse events are rare and the incidence of fatal complications are extremely low [32]. Nevertheless, despite the increasing availability of FMT in different countries [11], data on safety especially in the long-term follow-up are still highly lacking.

In this study we aimed to evaluate the primary efficacy of FMT treatment for recurrent CDI and factors associated with the failure of initial FMT treatment. Furthermore, we also aimed to investigate the peri-procedural safety profile of the nasoenteric FMT delivery method using a stringent safety protocol. Lastly, we wanted to evaluate the long-term efficacy and safety of FMT for recurrent CDI during a median follow up period of 24 months.

\section{METHODS}

Our study was based on a cohort of 60 consecutive patients who had undergone FMT for recurrent or refractory CDI. All patients for FMT procedure were referred to our centers. All patients who had undergone FMT in our center since $1^{\text {st }}$ of December 2015 were included in the study and were followed up until $1^{\text {st }}$ of September 2020.

\section{Recipients}

All 60 patients included in this study had a second or later episode of recurrent CDI or they failed to respond to the conventional treatment of the initial CDI. Clostridioides difficile infection diagnosis was confirmed by recurrent symptoms (diarrhea $>3$ times/day) and ELISA test by detecting enterotoxins A and B in patients' feces (Simple 2a-bdiff /stick 2a-bdiff, Operon, Spain). All patients had adequate treatment with oral vancomycin $500 \mathrm{mg}$ q.i.d for at least five days prior to the FMT procedure. Written informed consent was obtained from all the patients who participated in the study. The study was approved by the Regional Ethical Committee (Protocol No: BE-2-31).

\section{Donor Selection}

Donor screening protocol was performed based on the screening design of previously published standards $[10,33]$. Two donors unrelated to the patients, participated in the donation of feces to our center. Donors were healthy, younger than 35 years old with no risk factors and contraindications for feces donation. Before donating feces, the donors were confirmed to be without a history of using antibiotic therapy for at least six months prior to feces donation. They also did not have events of considerable infectious diseases 3 months before and during the participation in donating the feces. Donors had to undergo microbiological screening which consists of assessing blood for hepatitis A, B and C viruses, human immunodeficiency virus, Ebstein Barr virus, Cytomegalovirus, Treponema pallidum. Stools were assayed for standard pathogenic agents: Clostridioides difficile, enteric pathogens, including Salmonella, Shigella, Campylobacter, Yersinia, norovirus, Giardia lamblia and Criptosporidium parvum, protozoa and helminths.

\section{Preparation of Stool for Transplantation}

Fresh feces were used for FMT in the study. Feces were collected in special disposable containers and later stored in $4^{\circ} \mathrm{C}$ temperature until preparation but no longer than 6 hours. Preparation of FMT material was performed in fume cupboards. $50 \mathrm{~g}$ of fecal material was mixed with $150 \mathrm{ml}$ isotonic $0.9 \% \mathrm{NaCl}$ solution using a blender. The mixture was then filtered to remove solid mater and additional $0.9 \% \mathrm{NaCl}$ isotonic solution was added up to a total volume of $500 \mathrm{ml}$. The prepared FMT suspension was then transferred to the special bag that was later attached to 8 Fr nasoenteric tube (Kangaroo ${ }^{\text {Tm }}$ Nasogastric Feeding Tube, Cardinal Health, USA).

\section{Patient Preparation and FMT Procedure}

Prior to FMT all recipients had to complete at least five days treatment of oral vancomycin $500 \mathrm{mg}$ q.i.d. All the patients also received two doses of omeprazole $(40 \mathrm{mg}): 1$ ) in the evening before and 2) on the morning prior to FMT administration. Vancomycin was discontinued in the evening before the day of FMT administration. Transplantation of fecal microbiota for all 60 patients was performed via the nasoenteric tube which was placed into the descending duodenum during upper gastrointestinal endoscopy. To reduce the risk of aspiration and to make sure that the tube was positioned inside the duodenum, an abdominal X-ray was performed for all the patients after endoscopy to confirm correct positioning of the tube. Transplantation material was infused while patients were lying in the bed in a $45^{\circ}$ upright position. In order to prevent the aspiration of the transfused material, the patients had to stay in the same $45^{\circ}$ upright position for at least 4 hours after transplantation. With the aim to monitor recipients and avoid complications associated with the FMT, patients were under attendance of medical staff during the procedure and every 30 minutes, for six hours following FMT. After delivery of FMT, the nasoenteric tube was rinsed with $20 \mathrm{ml}$ of water before removal. If needed, a second or a third FMT procedure was performed using the same protocol using feces from the same donor as for initial FMT.

\section{Evaluation of Outcomes}

Resolution of the diarrhea was considered as an initial response to FMT therapy $[8,34,35]$. Primary non-responders were defined as patients who experienced failure within the 
first week of FMT including ongoing diarrhea after FMT. We have defined the cure of CDI as absence of diarrhea for 8 weeks $[34,35]$. Additional stool testing for Clostridioides difficile was not performed, as it is not recommended by guidelines [10]. Our patients were followed-up from the date of the procedure until $1^{\text {st }}$ of September 2020. Their health status was followed for at least 12 months after FMT with the longest follow-up period of 55 weeks. A minority of patients had outpatient visits and most patients received a telephone call by physicians. Data about early adverse events, including the appearance of abdominal pain, recurrent diarrhea, fever and any other new symptom, were collected. In the later follow up period, the questionnaire included questions about late adverse events, hospital admissions and newly occurred autoimmune, infectious, metabolic disease.

\section{Statistical Analysis}

Statistical analysis was performed with the SPSS 22.0 package. Descriptive statistics were presented as mean \pm standard deviation for continuous variables and as percentages for categorical variables.

\section{RESULTS}

Our study included 60 consecutive patients that were treated in our centers from 2015 to 2019 for recurrent or refractory CDI. The median patient age was 72.5 and the range was $32-99$ years. Twenty-eight females $(46.7 \%)$ and 32 males $(53.3 \%)$ were included in this study. The mean of previous CDI episodes before FMT was $2.7 \pm 1.3$ ranging from one to seven times. Nine $(15 \%)$ patients were under immunosuppressant therapy (glucocorticoids, azathioprine, methotrexate, tacrolimus or mycophenolate mofetil) and continued using these medications after FMT. Fifty patients (83.3\%) within our cohort had comorbidities, which were defined by the presence of two or more chronic diseases in a patient. Patients included in this study were treated with standard therapies using metronidazole, vancomycin before FMT and failed to show clinical improvement. Fecal microbiota transplantation was chosen as the treatment option for patients after the second or later CDI recurrence or for those who failed to respond to standard therapies.

Follow up data after FMTs included information about recurrent CDI episodes, early and late complications, health status at 3 and 12, 24 and 36 months after FMT where available and at the end of the follow-up period. Median follow-up time was 20 months (range: 1-55 months). Forty-eight out of 60 patients achieved full remission after the first FMT resulting in a $80 \%$ primary cure rate. Primary non-responders were defined as patients who experienced failure within the first week of FMT including ongoing diarrhea after FMT. Twelve patients had recurrent diarrhea after initial FMT, and they repeated FMT via the nasoenteric route after receiving oral vancomycin $500 \mathrm{mg}$ q.i.d. for at least five days once again. Ten of 12 patients responded to the second FMT with a full resolution of diarrhea.

In two patients, diarrhea persisted after the second FMT procedure. The first patient had type 2 diabetes, Parkinson's disease, stage 4 chronic kidney disease, chronic lung disease, senile myocardial infarction, hypothyroidism in his medical history. The second patient received broad spectrum antibiotics for pneumonia and skin infection treatment. For both patients FMT was performed for the third time and clinical remission was achieved resulting in a final overall cure rate of $100 \%$. All 12 recurrent CDI after first FMT occurred no longer than 7 weeks after initial FMT.

Follow up data and adverse events are presented in Table I. Three patients died within 8 weeks after FMT, but all these deaths were not FMT related. All three patients had comorbidities and fatal outcome was associated with existing chronic diseases. One patient was hospitalized due to ileus three weeks after FMT, but we could not confirm that it was related to FMT. This patient received conservative ileus treatment and fully recovered with the appropriate treatment.

Table I. Adverse events after fecal microbiota transplantation (FMT)

\begin{tabular}{lcc}
\hline Adverse event & N & $\%$ \\
\hline Periprocedural adverse events & & 0 \\
Regurgitation of donor feces or vomiting & 0 & 1.67 \\
Fever after FMT & 1 & 0 \\
Pneumonia after FMT & 0 & 0 \\
Endoscopy related events & 0 & \\
Adverse events at 12 weeks of follow-up & & 1.67 \\
Other probably FMT related events & 1 & 0 \\
Deaths (FMT related) & 0 & 2.5 \\
Deaths (not FMT related) & 3 & \\
Adverse events at the end of the follow-up period & & 0 \\
New onset oncologic diseases & 0 & 0 \\
New onset cardiovascular diseases & 0 & 5 \\
Deaths (not FMT related) & 6 & \\
\hline
\end{tabular}

After the FMT procedure a few minor adverse events were noted. Some patients reported nausea, slight abdominal discomfort, but these symptoms resolved within several hours after completion of FMT. There was one reported fever episode within 12 hours of FMT. Despite a single fever episode, response to treatment was adequate and no recurrent CDI occurred in this patient. During our study no SAEs was documented. Six patients died during the follow-up period due to severe comorbidities; all of them had a positive response to FMT.

We grouped initial responders and patients who recurred after the first FMT and analyzed their clinical data (Table II). No significant differences were found between these groups in terms of age, gender distribution, previous use of antibiotics, or use of immunosuppressant drugs. Distribution of people with comorbidities was similar in both groups (83.3\%).

\section{DISCUSSION}

This study confirms the excellent efficacy of FMT for recurrent and refractory CDI treatment. In the present study, the primary CDI cure rate was $80 \%$ and with repeatedly performed FMT all 60 patients achieved complete symptoms resolution. Within our cohort of patients, there was no significant difference between immunocompromised and 
Table II. Comparison of patient characteristics between initial responders and non-responders after first fecal microbiome transplantation for recurrent Clostridioides difficile infection (CDI)

\begin{tabular}{|c|c|c|c|}
\hline & Responder $(n=48)$ vs & Non-responders $(n=12)$ & $\mathrm{p}$ \\
\hline \multicolumn{4}{|l|}{ Age (years) } \\
\hline Median & 74 & 67.5 & \\
\hline Mean \pm SD & $71.8 \pm 12.9$ & $61.7 \pm 19.5$ & 0.124 \\
\hline Range & {$[37-99]$} & {$[32-85]$} & \\
\hline \multicolumn{4}{|l|}{ Gender, $\mathrm{n}(\%)$} \\
\hline Female & $27(56.3)$ & $6(50)$ & 0.697 \\
\hline \multicolumn{4}{|l|}{ Previous episodes of CDI } \\
\hline Mean \pm SD & $2.6 \pm 1.3$ & $2.6 \pm 1.3$ & 0.804 \\
\hline Range & {$[1-7]$} & {$[1-5]$} & \\
\hline Use of immunosuppressants, $\mathrm{n}(\%)$ & $6(12.5)$ & $3(25)$ & 0.365 \\
\hline Comorbidities, $\mathrm{n}(\%)$ & $40(83.3)$ & $10(83.3)$ & 1 \\
\hline
\end{tabular}

immunocompetent patient groups with respect to efficacy of FMT for recurrent CDI. Most importantly, our study demonstrates that FMT is a safe procedure with a very low risk both in the short and long-term follow up periods and enriches the area of knowledge related to FMT related adverse events where data are still scarce.

Systematic reviews and meta-analyses by Quraishi et al. [12] and lately Ianiro et al. [7] showed the high efficacy of FMTs for recurrent CDI administered by upper gastrointestinal route. Nevertheless, there are certain risk factors that are emerging as predictors of early or late treatment failure. A recently published study concluded that the use of non-CDI antibiotics, diagnosis of inflammatory bowel disease, severe CDI, poor quality of colonoscopy preparation and inpatient status can predict FMT failure [36]. In our cohort only two patients failed to achieve clinical remission after one or two FMT, while $80 \%$ were successfully treated just with one FMT procedure. Reduced efficacy of FMT could be associated with underlying comorbidities, immunosuppressant drugs, severe CDI, inpatient status, use of non-CDI antibiotics [36-39]. Previously published data also suggest that initial multiple infusions might increase FMT efficacy [7]. We did not observe significant differences when comparing the clinical characteristics of responders and non-responders, but this could be related to the sample size of the population.

There is a debate regarding the safety and effectiveness of FMT in immunocompromised patients. Earlier studies showed that FMT was equally effective for patients under immunosuppression therapy [40]. A later systematic review supports this statement, also suggesting that researchers did not observe increased rate of adverse events in immunosuppressive patients [41-43]. Small studies suggest that FMT have equal efficacy and is safe for cancer patients who undergone chemotherapy [44]. Nevertheless, Allegretti et al. [45] identified immunosuppressive therapy as risk factor for FMT failure then multiple FMTs were performed for recurrent CDI disease. From our data FMT efficacy and safety for immunosuppressed patients was equal to immunocompetent patients, but we are aware that only nine patients with immunosuppression therapy were included. Despite good initial results further larger studies are needed to evaluate FMT therapy safety and efficacy for immunosuppressed patients.
As a general rule, short-term SAEs after FMT are rare and mostly procedure related. Complications of FMT administration via upper gastrointestinal route are most often related to material regurgitation which can lead to aspiration pneumonia and death $[32,46]$. In order to prevent procedure related SAEs, we followed all the patients with very stringent peri-procedural follow-up protocol that is described in the method section. We believe that these measures may help to avoid procedure related complications when FMT is administered via the nasoenteric route. Emerging studies showed excellent FMT safety and low SAE rates for both upper and lower gastrointestinal transplant delivery routes [31, 47]. Saha et al. [48] has recently published a prospective study that included 609 patients and their follow-up data showed that FMT was safe and was associated with very low rates of SAEs. Nevertheless, FMT still lacks prospective efficacy and safety information [49]. In our study 20 patients were followed for up to 36 months and no SAEs or FMT related deaths were documented. It is worth pointing out the remaining concerns about infectious disease transmission. Several case reports suggested that enteric pathogens were transmitted through the donor feces $[50,51]$. These patients have developed Escherichia coli infections and three reported deaths could be related to FMT transmitted infection as issued by the Food Drug Administration (FDA) [51-53]. For this reason, a longterm and in-depth follow up are highly indicated to evaluate the possibility for transfer of infections, chronic diseases or alterations of gut microbiota.

Bowel lavage is recommended by guidelines for both upper and lower gastrointestinal routes in preparation for FMT [10]. However, there is not enough data to confirm its benefits when transplantation is performed via the upper gastrointestinal route [30]. Several studies show similar efficacy results without bowel preparation compared to studies when bowel lavage was used [12, 33, 46]. For this reason, bowel preparation was not used because it greatly increases patients' comfort, and we did not observe decreased efficacy in this study. A recent systematic review and meta-analysis identified poor bowel preparation as a risk factor for FMT failure when lower gastrointestinal route was chosen [36]. Further studies are welcome to properly evaluate bowel preparation efficacy for FMT via upper gastrointestinal route. 
This study has several limitations that need to be acknowledged. The results of the study and subgroup analyses might be limited by the relatively small sample size. Furthermore, complete data on clinical characteristics was not available for all patients because many of them were referred for FMT from other clinical centers. Our study included patients with FMT since the year 2015 and the cohort included the use of fresh feces. We want to emphasize that our center currently uses only frozen fecal material for FMT and tests them for multi-drug resistant bacteria including extended spectrum beta-lactamase producing Enterobacteriaceae (ESBL), vancomycin-resistant enterococci (VRE), carbapenemresistant Enterobacteriaceae (CRE), and methicillin-resistant Staphylococcus aureus (MRSA) and such practice should be followed by other centers as suggested currently in the available guidelines $[29,49]$. Furthermore, additional testing for COVID-19 is mandatory and should be implemented due to the world-wide pandemic situation [54]. We also admit that the administration of FMT via colonoscopy might have slightly higher efficacy results and the choice of FMT administration should be carefully considered in each center.

\section{CONCLUSIONS}

Our study confirms excellent rates of FMT efficacy in the treatment of recurrent CDI. In addition, this study shows that it is possible to avoid short term SAEs when FMT is administered via the nasoenteric tube by following a very stringent peri-procedural patient follow-up protocol. Our study also demonstrates good safety with low risk of long adverse events after FMT.

Conflict of interest: None to declare.

Authors' contributions: T.U., G.I., A.G., J.K. prepared the concept and design of the study. All authors contributed to the data acquisition. T.U. and G.I. performed the statistical analyses, interpreted the results and drafted the manuscript. All authors critically revised the manuscript, approved the final version to be published, and agree to be accountable for all aspects of the work.

\section{REFERENCES}

1. Pilcante J, Rojas P, Ernst D, et al. Clostridium difficile infection in Chilean patients submitted to hematopoietic stem cell transplantation. Rev Bras Hematol Hemoter 2015;37:388-394. doi:10.1016/j. bjhh.2015.07.010

2. Abou Chakra CN, McGeer A, Labbe AC, et al. Factors Associated with Complications of Clostridium difficile Infection in a Multicenter Prospective Cohort. Clin Infect Dis 2015;61:1781-1788. doi:10.1093/ cid/civ749

3. Revolinski S. Implementation of a clinical decision support alert for the management of Clostridium difficile infection. Antibiotics (Basel) 2015;4:667-674. doi:10.3390/antibiotics4040667

4. Ianiro G, Bibbò S, Gasbarrini A, Cammarota G. Therapeutic Modulation of Gut Microbiota: Current Clinical Applications and Future Perspectives. Curr Drug Targets 2014;15:762-770. doi:10.2174/13894 50115666140606111402
5. Smits WK, Lyras D, Lacy DB, Wilcox MH, Kuijper EJ. Clostridium difficile infection. Nat Rev Dis Prim 2016;2:16020. doi:10.1038/ nrdp. 2016.20

6. Cammarota G, Ianiro G, Magalini S, Gasbarrini A, Gui D. Decrease in surgery for clostridium difficile infection after starting a program to transplant fecal microbiota. Ann Intern Med 2015;163:487-478. doi:10.7326/L15-5139

7. Ianiro G, Maida M, Burisch J, et al. Efficacy of different faecal microbiota transplantation protocols for Clostridium difficile infection: A systematic review and meta-analysis. United Eur Gastroenterol J 2018;6:1232-1244. doi:10.1177/2050640618780762

8. Mullish BH, Quraishi MN, Segal JP, et al. The use of faecal microbiota transplant as treatment for recurrent or refractory Clostridium difficile infection and other potential indications: Joint British Society of Gastroenterology (BSG) and Healthcare Infection Society (HIS) guidelines. Gut 2018;67:1920-1941. doi:10.1136/ gutjnl-2018-316818

9. Hvas CL, Dahl Jørgensen SM, Jørgensen SP, et al. Fecal Microbiota Transplantation Is Superior to Fidaxomicin for Treatment of Recurrent Clostridium difficile Infection. Gastroenterology 2019;156:1324-1332. e3. doi:10.1053/j.gastro.2018.12.019

10. Cammarota G, Ianiro G, Tilg H, et al. European consensus conference on faecal microbiota transplantation in clinical practice. Gut 2017;66:569580. doi:10.1136/gutjnl-2016-313017

11. Baunwall SMD, Terveer EM, Dahlerup JF, et al. The use of Faecal Microbiota Transplantation (FMT) in Europe: A Europe-wide survey. Lancet Reg Heal Eur 2021;100181. doi:10.1016/j.lanepe.2021.100181

12. Quraishi MN, Widlak M, Bhala N, et al. Systematic review with meta-analysis: the efficacy of faecal microbiota transplantation for the treatment of recurrent and refractory Clostridium difficile infection. Aliment Pharmacol Ther 2017;46:479-493. doi:10.1111/apt.14201

13. Hocquart M, Lagier JC, Cassir N, et al. Early fecal microbiota transplantation improves survival in severe clostridium difficile infections. Clin Infect Dis 2018;66:645-650. doi:10.1093/cid/cix762

14. Heimann SM, Cruz Aguilar MR, Mellinghof S, Vehreschild MJGT. Economic burden and cost-effective management of Clostridium difficile infections. Med Mal Infect 2018;48:23-29. doi:10.1016/j. medmal.2017.10.010

15. Paramsothy S, Paramsothy R, Rubin DT, et al. Faecal microbiota transplantation for inflammatory bowel disease: A systematic review and meta-analysis. J Crohn's Colitis 2017;11:1180-1199. doi:10.1093/ ecco-jcc/jjx063

16. Galazzo G, Tedjo DI, Wintjens DSJ, et al. Faecal Microbiota Dynamics and their Relation to Disease Course in Crohn's Disease. J Crohns Colitis 2019;13:1273-1282. doi:10.1093/ecco-jcc/jjz049

17. Paramsothy S, Kamm MA, Kaakoush NO, et al. Multidonor intensive faecal microbiota transplantation for active ulcerative colitis: a randomised placebo-controlled trial. Lancet 2017;389:1218-1228. doi:10.1016/S0140-6736(17)30182-4

18. Meighani A, Hart BR, Bourgi K, Miller N, John A, Ramesh M. Outcomes of Fecal Microbiota Transplantation for Clostridium difficile Infection in Patients with Inflammatory Bowel Disease. Dig Dis Sci 2017;62:28702875. doi:10.1007/s10620-017-4580-4

19. Myneedu K, Deoker A, Schmulson MJ, Bashashati M. Fecal microbiota transplantation in irritable bowel syndrome: A systematic review and meta-analysis. United European Gastroenterol J 2019;7:1033-1041. doi:10.1177/2050640619866990

20. El-Salhy M, Hatlebakk JG, Gilja OH, Bråthen Kristoffersen A, Hausken T. Efficacy of faecal microbiota transplantation for patients with irritable 
bowel syndrome in a randomised, double-blind, placebo-controlled study. Gut. 2020;69:859-867. doi:10.1136/gutjnl-2019-319630

21. Ianiro G, Eusebi LH, Black CJ, Gasbarrini A, Cammarota G, Ford AC. Systematic review with meta-analysis: efficacy of faecal microbiota transplantation for the treatment of irritable bowel syndrome. Aliment Pharmacol Ther 2019;50:240-248. doi:10.1111/apt.15330

22. Bajaj JS, Fagan A, Gavis EA, Kassam Z, Sikaroodi M, Gillevet PM. Long-term Outcomes of Fecal Microbiota Transplantation in Patients With Cirrhosis. Gastroenterology 2019;156:1921-1923.e3. doi:10.1053/j. gastro.2019.01.033

23. Alsahhar JS, Rahimi RS. Updates on the pathophysiology and therapeutic targets for hepatic encephalopathy. Curr Opin Gastroenterol 2019;35:145-154. doi:10.1097/MOG.0000000000000527

24. Ianiro G, Rossi E, Thomas AM, et al. Faecal microbiota transplantation for the treatment of diarrhoea induced by tyrosine-kinase inhibitors in patients with metastatic renal cell carcinoma. Nat Commun 2020;11:4333. doi:10.1038/s41467-020-18127-y

25. Wortelboer K, Nieuwdorp M, Herrema H. Fecal microbiota transplantation beyond Clostridioides difficile infections. EBioMedicine 2019;44:716-729. doi:10.1016/j.ebiom.2019.05.066

26. Chen D, Wu J, Jin D, Wang B, Cao H. Fecal microbiota transplantation in cancer management: Current status and perspectives. Int J Cancer 2019;145:2021-2031. doi:10.1002/ijc.32003

27. Vendrik KEW, Ooijevaar RE, de Jong PRC, et al. Fecal Microbiota Transplantation in Neurological Disorders. Front Cell Infect Microbiol 2020;10:98. doi:10.3389/fcimb.2020.00098

28. Morkūnas E, Skiecevičienė J, Kupčinskas J. The impact of modulating the gastrointestinal microbiota in cancer patients. Best Pract Res Clin Gastroenterol 2020;48-49:101700. doi:10.1016/j.bpg.2020.101700

29. Keller JJ, Ooijevaar RE, Hvas CL, et al. A standardised model for stool banking for faecal microbiota transplantation: a consensus report from a multidisciplinary UEG working group. United Eur Gastroenterol J 2021;9:229-247. doi:10.1177/2050640620967898

30. Bunnik EM, Aarts N, Chen LA. Physicians Must Discuss Potential LongTerm Risks of Fecal Microbiota Transplantation to Ensure Informed Consent. Am J Bioeth 2017;17:61-63. doi:10.1080/15265161.2017.1299816

31. Jalanka J, Hillamaa A, Satokari R, Mattila E, Anttila VJ, Arkkila P. The long-term effects of faecal microbiota transplantation for gastrointestinal symptoms and general health in patients with recurrent Clostridium difficile infection. Aliment Pharmacol Ther 2018;47:371379. doi:10.1111/apt.14443

32. Dailey FE, Turse EP, Daglilar E, Tahan V. The dirty aspects of fecal microbiota transplantation: a review of its adverse effects and complications. Curr Opin Pharmacol 2019;49:29-33. doi:10.1016/j. coph.2019.04.008

33. Van Nood E, Vrieze A, Nieuwdorp M, et al. Duodenal infusion of donor feces for recurrent clostridium difficile. N Engl J Med 2013;68:407-415 doi:10.1056/NEJMoa1205037

34. Surawicz CM, Brandt LJ, Binion DG, et al. Guidelines for diagnosis, treatment, and prevention of clostridium difficile infections. Am J Gastroenterol 2013;108:478-498. doi:10.1038/ajg.2013.4

35. Debast SB, Bauer MP, Kuijper EJ; European Society of Clinical Microbiology and Infectious Diseases. European society of clinical microbiology and infectious diseases: Update of the treatment guidance document for Clostridium difficile infection. Clin Microbiol Infect 2014;20 Suppl 2:1-26. doi:10.1111/1469-0691.12418

36. Tariq R, Hayat M, Pardi D, Khanna S. Predictors of failure after fecal microbiota transplantation for recurrent Clostridioides difficile infection: a systematic review and meta-analysis. Eur J Clin Microbiol Infect Dis 2021;40:1383-1392. doi:10.1007/s10096-021-04163-Z

37. Fischer M, Kao D, Mehta SR, et al. Predictors of Early Failure after Fecal Microbiota Transplantation for the Therapy of Clostridium Difficile Infection: A Multicenter Study. Am J Gastroenterol 2016;111:1024-1031. doi:10.1038/ajg.2016.180

38. Meighani A, Hart BR, Mittal C, Miller N, John A, Ramesh M. Predictors of fecal transplant failure. Eur J Gastroenterol Hepatol 2016;28:826-830. doi:10.1097/MEG.0000000000000614

39. Ianiro G, Valerio L, Masucci L, et al. Predictors of failure after single faecal microbiota transplantation in patients with recurrent Clostridium difficile infection: results from a 3-year, singlecentre cohort study. Clin Microbiol Infect 2017;23:337.e1-337.e3. doi:10.1016/j.cmi.2016.12.025

40. Kelly CR, Ihunnah C, Fischer M, et al. Fecal microbiota transplant for treatment of clostridium difficile infection in immunocompromised patients. Am J Gastroenterol 2014;109:1065-1071. doi:10.1038/ ajg.2014.133

41. Di Bella S, Gouliouris T, Petrosillo N. Fecal microbiota transplantation (FMT) for Clostridium difficile infection: Focus on immunocompromised patients. J Infect Chemother 2015;21:230-237. doi:10.1016/j. jiac.2015.01.011

42. Ianiro G, Maida M, Burisch J, et al. Efficacy of different faecal microbiota transplantation protocols for Clostridium difficile infection: A systematic review and meta-analysis. United European Gastroenterol J 2018;6:1232-1244. doi:10.1177/2050640618780762

43. Shogbesan O, Poudel DR, Victor S, et al. A Systematic Review of the Efficacy and Safety of Fecal Microbiota Transplant for Clostridium difficile Infection in Immunocompromised Patients. Can J Gastroenterol Hepatol 2018;2018:1394379. doi:10.1155/2018/1394379

44. Cammarota G, Ianiro G. Gut Microbiota and Cancer Patients: A Broad-Ranging Relationship. Mayo Clin Proc 2017;92:1605-1607. doi:10.1016/j.mayocp.2017.09.009

45. Allegretti JR, Mehta SR, Kassam Z, et al. Risk Factors that Predict the Failure of Multiple Fecal Microbiota Transplantations for Clostridioides difficile Infection. Dig Dis Sci 2021;66:213-217. doi:10.1007/s10620020-06198-2

46. van Beurden YH, de Groot PF, van Nood E, Nieuwdorp M, Keller JJ, Goorhuis A. Complications, effectiveness, and long term followup of fecal microbiota transfer by nasoduodenal tube for treatment of recurrent Clostridium difficile infection. United European Gastroenterol J 2017;5:868-879. doi:10.1177/2050640616678099

47. Kelly CR, Yen EF, Grinspan AM, et al. Fecal Microbiota Transplantation Is Highly Effective in Real-World Practice: Initial Results From the FMT National Registry. Gastroenterology 2021;160:183-192.e3. doi:10.1053/j. gastro.2020.09.038

48. Saha S, Mara K, Pardi DS, Khanna S. Long-term Safety of Fecal Microbiota Transplantation for Recurrent Clostridioides difficile Infection. Gastroenterology 2021;160:1961-1969.e3. doi:10.1053/j. gastro.2021.01.010

49. Cammarota G, Ianiro G, Kelly CR, et al. International consensus conference on stool banking for faecal microbiota transplantation in clinical practice. Gut 2019;68:2111-2121. doi:10.1136/ gutjnl-2019-319548

50. Zellmer C, Sater MRA, Huntley MH, Osman M, Olesen SW, Ramakrishna B. Shiga Toxin-Producing Escherichia coli Transmission via Fecal Microbiota Transplant. Clin Infect Dis 2021;72:e876-e880. doi:10.1093/cid/ciaal486 
51. DeFilipp Z, Bloom PP, Torres Soto M, et al. Drug-Resistant E. coli Bacteremia Transmitted by Fecal Microbiota Transplant. N Engl J Med 2019;381:2043-2050. doi:10.1056/NEJMoa1910437

52. FDA. Fecal Microbiota for Transplantation: Safety Alert - Risk of Serious Adverse Events Likely Due to Transmission of Pathogenic Organisms. [Cited 2021 Feb 5]. Available from: https://www.fda. gov/safety/medical-product-safety-information/fecal-microbiotatransplantation-safety-alert-risk-serious-adverse-events-likely-duetransmission
53. FDA News Alerts. Fecal Microbiota for Transplantation : Safety Communication- Risk of Serious Adverse Reactions Due to Transmission of Multi-Drug Resistant Organisms. 2019. Available from: https://www.pharmareviews.in/fecal-microbiota-for-transplantationsafety-communication-risk-of-serious-adverse-reactions-due-totransmission-of-multi-drug-resistant-organisms/

54. Ianiro G, Mullish BH, Hvas CL, et al. SARS-CoV-2 vaccines and donor recruitment for FMT. Lancet Gastroenterol Hepatol 2021;6:264-266. doi:10.1016/S2468-1253(21)00032-7 